University of Nebraska - Lincoln

DigitalCommons@University of Nebraska - Lincoln

6-2009

\title{
PHOTOMETRY OF TYPE II CEPHEID CANDIDATES FROM THE NORTHERN PART OF THE ALL SKY AUTOMATED SURVEY
}

\author{
Edward G. Schmidt \\ University of Nebraska-Lincoln, eschmidt1@unl.edu \\ Brian Hemen \\ University of Nebraska - Lincoln, bhemen1@bigred.unl.edu \\ Danielle Rogalla \\ University of Nebraska - Lincoln, drogall1@bigred.unl.edu \\ Lauren Thacker-Lynn \\ University of Nebraska - Lincoln, Ithacke1@bigred.unl.edu \\ University of Nebraska - Lincoln
}

Follow this and additional works at: https://digitalcommons.unl.edu/physicsschmidt

Part of the Physics Commons

Schmidt, Edward G.; Hemen, Brian; Rogalla, Danielle; Thacker-Lynn, Lauren; and University of Nebraska Lincoln, "PHOTOMETRY OF TYPE II CEPHEID CANDIDATES FROM THE NORTHERN PART OF THE ALL SKY AUTOMATED SURVEY" (2009). Edward Schmidt Publications. 35.

https://digitalcommons.unl.edu/physicsschmidt/35

This Article is brought to you for free and open access by the Research Papers in Physics and Astronomy at DigitalCommons@University of Nebraska - Lincoln. It has been accepted for inclusion in Edward Schmidt Publications by an authorized administrator of DigitalCommons@University of Nebraska - Lincoln. 


\title{
PHOTOMETRY OF TYPE II CEPHEID CANDIDATES FROM THE NORTHERN PART OF THE ALL SKY AUTOMATED SURVEY
}

\author{
Edward G. Schmidt, Brian Hemen, Danielle Rogalla, and Lauren Thacker-Lynn \\ Department of Physics and Astronomy, University of Nebraska, Lincoln, NE, USA; eschmidt1@ unl.edu, bhemen1@bigred.unl.edu, drogall1 @ bigred.unl.edu, \\ lthacke1@ bigred.unl.edu \\ Received 2008 December 10; accepted 2009 February 28; published 2009 April 21
}

\begin{abstract}
We have obtained $V R$ photometry of 282 Cepheid variable star candidates from the northern part of the All Sky Automated Survey (ASAS). These together with data from the ASAS and the Northern Sky Variability Survey (NSVS) were used to redetermine the periods of the stars. We divided the stars into four groups based on location in a plot of mean color, $\langle V-R\rangle$, versus period. Two of the groups fell within the region of the diagram containing known type II Cepheids and yielded 14 new highly probable type II Cepheids. The properties of the remaining stars in these two groups are discussed but their nature remains uncertain. Unexplained differences exist between the sample of stars studied here and a previous sample drawn from the NSVS by Akerlof et al. This suggests serious biases in the identification of variables in different surveys.
\end{abstract}

Key words: Cepheids - stars: Population II

Online-only material: machine-readable and VO tables

\section{INTRODUCTION}

We have undertaken a search for new type II Cepheid variable stars based on the results of recent large area surveys. The goal is to greatly increase the number of such stars available for a variety of astrophysical investigations including studies of the structure of the galactic thick disk and halo, stellar pulsation, and the late stages of stellar evolution. See Schmidt et al. (2007, Paper I) for further discussion of the background of this project.

Paper I presented photometry of 205 Cepheid candidates discovered by Akerlof et al. (2000) in the ROTSE-I demonstration project. Based on the photometric properties, we concluded that 97 were likely type II Cepheids and 17 were possible type II Cepheids. Thus, the photometry proved useful for screening out a significant number of candidates. However, the amplitude and period distributions of these stars differed significantly from known type II Cepheids, leaving their status in doubt. The present paper continues this work by presenting photometry for Cepheid candidates from the northern part of the All Sky Automated Survey (ASAS; Pojmanski et al. 2005).

\section{THE DATA}

\subsection{Sample Selection}

The northern ASAS catalog contains 11,509 variables ranging from $V=5.6$ to 14.3 . For most right ascensions, the variables are located between declinations of $-4^{\circ}$ and $+28^{\circ} .5$. However, between $14^{\mathrm{h}} .0$ and $15^{\mathrm{h}} .8$ and between $17^{\mathrm{h}} .3$ and $24^{\mathrm{h}} .0$, there are no stars north of 20.5. Similarly, no stars are found in the catalog south of $0^{\circ}$ for right ascensions larger than $18^{\mathrm{h}} .0$.

In the electronic version of the catalog, Pojmanski et al. (2005) list one or more possible classifications for each star based on their photometry combined with infrared data from the Two Micron All Sky Survey. We selected those stars for which at least one possible classification was as a Cepheid (DCEP or CW in the notation of Pojmanski et al. 2005). We then removed stars with periods outside of the range from 1 to 100 days and stars which were identified with known variables in the General Catalog of Variable Stars
(GCVS). Finally, since our main interest is in identifying type II Cepheids, we removed stars closer than $10^{\circ}$ from the Galactic plane. This resulted in a sample of 321 stars. No stars were removed on the basis of amplitude since our previous study described in Paper I revealed many interesting stars with small amplitudes.

The Hubble Space Telescope Guide Star Catalog was used to select comparison stars for the variables. In the process, 39 stars were found to be too bright to observe with our instrumentation or lacked suitable comparison stars within the field of our CCD. Thus, the final sample presented here consists of 282 stars.

The final program stars that were observed are listed in Table 1. The first column contains a running number for use in this paper, and Column 2 gives the star designation from the ASAS. Columns 3, 4, and 5 give the number of photometric observations used for each star from the Northern Sky Variability Survey (NSVS; Wozniak et al. 2004), the ASAS, and the present study, respectively.

To search for selection biases, we plotted various parameters for our program stars against galactic latitude and longitude. These included the periods and amplitudes from both Pojmanski et al. (2005) and from the present study (Table 3) as well as the mean colors and magnitudes from Table 3. We were unable to discern any dependence of these quantities on position. We conclude that our sample is reasonably homogeneous in the region of the sky it covers.

\subsection{The Observations}

We made about $6600 R V$ observations of the Cepheid candidates between 2005 August and 2008 July (JD 2,453,597 and $2,454,661)$ at Behlen Observatory. The same instrumentation and procedures for collecting and processing the data were used as described in Paper I and the reader is referred to that publication for details. The individual Behlen observations are listed in Table 2.

When it became clear from our observations that a particular star was too red to be a possible type II Cepheid, it was dropped from the program. This accounts for a dozen or so stars with fewer than 10 observations. 
Table 1

Program Stars

\begin{tabular}{|c|c|c|c|c|}
\hline $\begin{array}{l}\text { No. } \\
\text { (1) } \\
\end{array}$ & $\begin{array}{c}\text { ASAS } \\
\text { Designation } \\
\text { (2) }\end{array}$ & $\begin{array}{c}N_{\text {obs }} \\
\text { (NSVS) } \\
(3)\end{array}$ & $\begin{array}{c}N_{\text {obs }} \\
(\text { ASAS) } \\
(4)\end{array}$ & $\begin{array}{c}N_{\text {obs }} \\
\text { (Behlen) } \\
(5)\end{array}$ \\
\hline A001 & $000606+0345.1$ & 192 & 95 & 28 \\
\hline $\mathrm{A} 002$ & $000801+1323.3$ & 133 & 59 & 29 \\
\hline A003 & $000906+1659.8$ & 181 & 62 & 27 \\
\hline A004 & $001430+1331.1$ & 108 & 74 & 22 \\
\hline $\mathrm{A} 005$ & $001729+1208.1$ & 116 & 98 & 33 \\
\hline
\end{tabular}

Note. (This table is available in its entirety in machine-readable and Virtual Observatory (VO) forms in the online journal. A portion is shown here for guidance regarding its form and content.)

Table 2

Photometric Data

\begin{tabular}{lccc}
\hline \hline Star & HJD & $V$ & $V-R$ \\
& $-2,450,000$ & & $(4)$ \\
$(1)$ & $(2)$ & $(3)$ & 0.567 \\
\hline A001 & 3615.727 & 13.008 & 0.574 \\
A001 & 3616.781 & 13.009 & 0.568 \\
A001 & 3617.790 & 13.003 & 0.604 \\
A001 & 3627.676 & 13.056 & 0.585 \\
A001 & 3630.780 & 13.028 & \\
\hline
\end{tabular}

Note. (This table is available in its entirety in machine-readable and Virtual Observatory (VO) forms in the online journal. A portion is shown here for guidance regarding its form and content.)

For our analysis, we have combined our observations with those from the ASAS and the NSVS. Since the NSVS photometry is not on the standard $V$ system, we added a zero-point correction, determined individually for each star, to bring the average magnitude into agreement with the Behlen $V$ observations. The three data sets together provide photometry for most of the stars every year from 1999 to 2007 . Such a long, reasonably continuous set of observations improves period de- terminations by suppressing aliases. It also allows the identification of irregular behavior or variations in the mean magnitudes, amplitudes, or light curve shapes over intervals of several years.

\subsection{Photometric Properties}

Table 3 contains the derived photometric properties of our program stars. Column 1 identifies the star with the running number from Table 1, while the period and its uncertainty (in units of the least significant figure of the cited period) are in Columns 2 and 3.

For each star, we searched for periods from 0.1 days to the Nyquist period using the data-compensated discrete Fourier transform method of Ferraz-Mello (1981) or the phase dispersion minimization method of Stellingwerf (1978). If they were of adequate quality, all three of the data sets were used. We then adjusted the period to optimally match the minima and maxima of the Behlen data to those of an earlier data set, generally the NSVS data since it provides the longest baseline. The uncertainty was determined by increasing and decreasing the adopted period until the match was unacceptable. Thus, the tabulated uncertainties represent upper limits. This, of course, assumes that we have selected the right peak among any aliases that are present in the power spectrum. The problem of aliasing is commonly encountered but is especially difficult in cases with large scatter, small amplitude, variable period, long-term variations, or small data sets. Some of these issues affect many of our stars.

The mean magnitudes often vary over intervals that are much longer than the primary period (indicated by footnote "d" to Table 3). When this was the case, the long-term variation was removed before the period search was conducted.

When a period could not be found due to period changes, irregularity, or problems with some of the data, no uncertainty is listed. In such cases, the tabulated period is the best estimate and is often merely the timescale of the variation.

Columns 4, 5, 6, and 7 of Table 3 list the intensity mean magnitudes and colors, $\langle V\rangle$ and $\langle V-R\rangle$, and their standard deviations. These parameters and those in the remaining columns

Table 3

Light-Curve Parameters

\begin{tabular}{|c|c|c|c|c|c|c|c|c|c|c|c|c|}
\hline $\begin{array}{l}\text { No. } \\
\text { (1) }\end{array}$ & $\begin{array}{l}\text { Period } \\
\text { (2) }\end{array}$ & $\begin{array}{l}\sigma_{P} \\
(3)\end{array}$ & $\begin{array}{r}\langle V\rangle \\
(4)\end{array}$ & $\begin{array}{l}\sigma_{V} \\
(5)\end{array}$ & $\begin{array}{c}\langle V-R\rangle \\
\text { (6) }\end{array}$ & $\begin{array}{c}\sigma_{V-R} \\
(7)\end{array}$ & $\begin{array}{l}\Delta V \\
(8) \\
\end{array}$ & $\begin{array}{l}\sigma_{l c} \\
(9)\end{array}$ & $\begin{array}{c}S \\
(10) \\
\end{array}$ & $\begin{array}{c}\sigma_{S} \\
(11)\end{array}$ & $\begin{array}{c}\text { Notes }^{\mathrm{a}} \\
(12)\end{array}$ & $\begin{array}{c}\text { Group }^{\mathrm{b}} \\
(13) \\
\end{array}$ \\
\hline A001 & 53 & $\ldots$ & 12.94 & 0.01 & 0.57 & 0.00 & 0.12 & 0.033 & 0.12 & 0.04 & $\mathrm{c}, \mathrm{d}, \mathrm{e}, \mathrm{g}$ & 1 \\
\hline A002 & 14.545 & 5 & 10.92 & 0.01 & 0.63 & 0.00 & 0.14 & 0.024 & 0.13 & 0.03 & $f, g$ & 1 \\
\hline A003 & 25.5 & $\ldots$ & 12.2 & $\cdots$ & 1.34 & $\ldots$ & 0.4 & $\ldots$ & 0.28 & 0.04 & e,IR & \\
\hline A004 & 9.897 & 5 & 12.13 & 0.01 & 0.77 & 0.00 & 0.86 & 0.037 & 0.09 & 0.01 & & (1) \\
\hline A005 & 4.4148 & 3 & 12.23 & 0.01 & 0.65 & 0.01 & 0.14 & 0.035 & 0.14 & 0.02 & $\mathrm{c}, \mathrm{d}, \mathrm{f}, \mathrm{g}$ & 1 \\
\hline
\end{tabular}

Notes.

a The lowercase letters refer to footnotes. The uppercase codes indicate reasons for rejecting the star as a Cepheid candidate: CO, constant star; EC, light curve indicates an eclipsing binary; IR, irregularity of the period or light curve eliminates the star as a Cepheid; SL, the slope of the color-magnitude relation falls outside of the expected range for a Cepheid; SP, period too short for a Cepheid

b The group in Figure 1(b) to which the star belongs, if any.

$c$ The amplitude varies.

$\mathrm{d}$ Variation in the mean magnitude.

e No period satisfies all of the data. The best period for the Behlen data is tabulated.

${ }^{\mathrm{f}}$ See the Appendix for a note regarding this star.

$\mathrm{g}$ The shape of the light curve varies or there is phase jitter present.

${ }^{\mathrm{h}}$ No period fits all of the data but there are too few data from Behlen to determine a period. The overall best period is tabulated and was used in the analysis.

${ }^{\mathrm{i}}$ No period satisfies all of the data. The period was derived from the Behlen and the NSVS data only.

j No period satisfies all of the data. The period was determined from the ASAS and Behlen data only.

(This table is available in its entirety in machine-readable and Virtual Observatory (VO) forms in the online journal. A portion is shown here for guidance regarding its form and content.) 

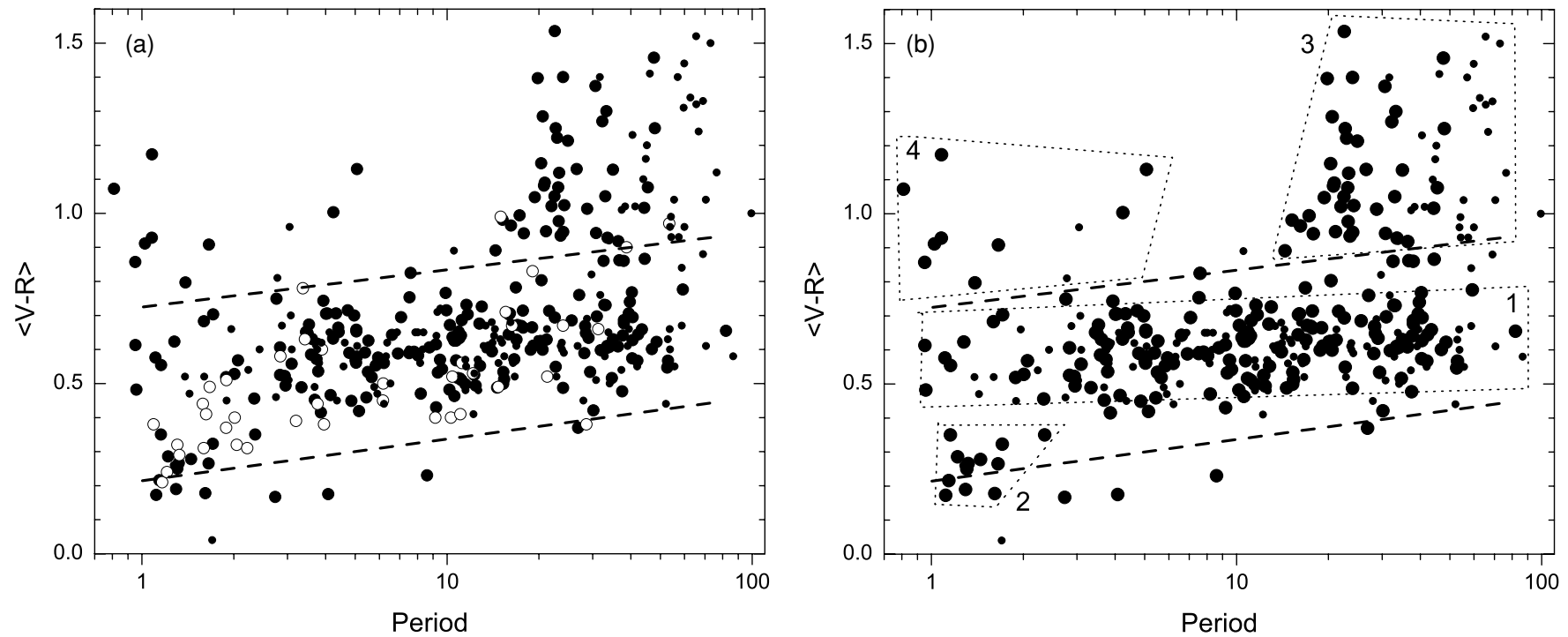

Figure 1. Mean colors plotted against period. (a) Large filled circles represent stars from the present sample that were not deleted as discussed in the text. Small circles represent stars from Paper I which were considered possible Cepheid candidates as well as stars that were rejected for being too red. Open circles represent known Cepheids and the dashed lines delineate the region they occupy. (b) The same diagram with the known Cepheids omitted to reduce crowding and with regions shown for the groups of stars discussed in the text.

were determined from the Behlen data only. The standard deviations were calculated as described in Paper I. When no period could be determined for a star or when the data were too poorly distributed in phase to determine a reliable mean, the magnitudes and colors are simple arithmetic means of all the data points and no error is listed. For some eclipsing binaries, particularly those with narrow eclipses, there are too few magnitudes during eclipse to determine a period. In those cases, $\langle V\rangle$ and $\langle V-R\rangle$ were determined from the data outside of eclipse when the star is generally relatively constant.

For the majority of stars a well-determined Fourier series, usually of order 1 or 2 , could be fit to the data. The amplitude from this fit is given in Column 8 and the scatter (standard deviation) about the fit is listed in Column 9. A steep rise to maximum light can result in a poor Fourier fit. In a few such cases, the tabulated amplitude was adjusted and the poorly fit portions of the light curve were excluded from the determination of the scatter. For eclipsing binaries, stars without a reliable period and stars with insufficient phase coverage, the amplitudes are just the range of all the data points. Such values can be identified because no scatter is listed in Column 9. In most cases where the light curve scatter is larger than normal, an examination of the data indicates that it is caused by the lack of repetition of the light curve from one cycle to another.

Columns 10 and 11 list the slope of the color-magnitude trajectory for each star and its uncertainty.

Column 12 contains status flags and references to footnotes. Following Paper I, we have deleted stars from consideration as Cepheid candidates on the basis of inappropriate light curve form, negative slope of the color-magnitude trajectory, or short period (flags CO, EC, IR, SL, SP). Lowercase letters refer to the footnotes. Footnote "f" indicates that comments regarding individual stars can be found in the Appendix. Footnotes "c," "d," "e," "g," "h," "i," and "j" identify instabilities in the photometric behavior. Given the amount of data we have, it is sometimes difficult to distinguish changes in light curve shape from phase jitter so footnote " $\mathrm{g}$ " includes both possibilities.

Since the possible instabilities were based on a comparison of three independent data sets, in some cases they may

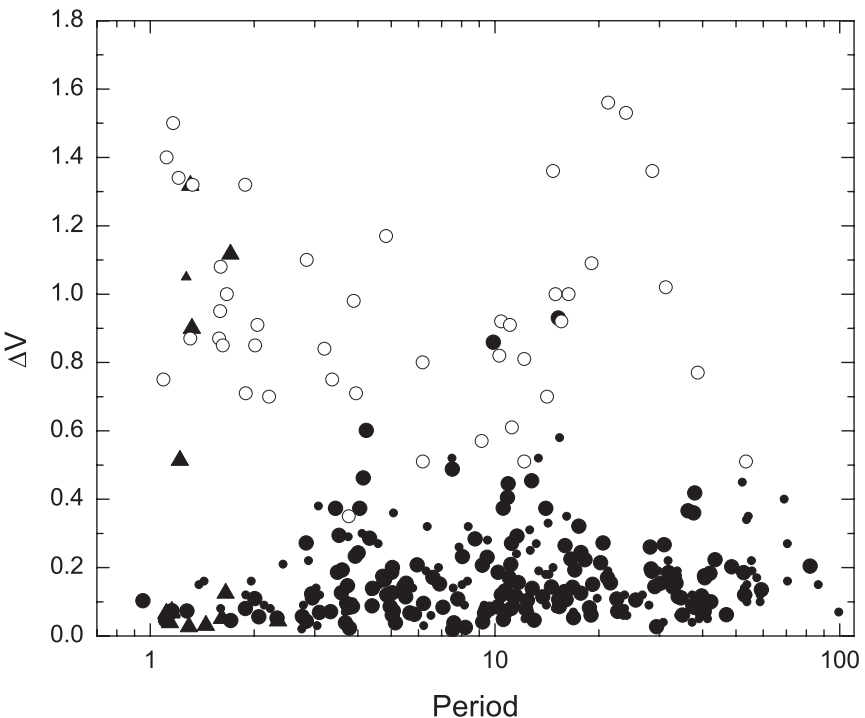

Figure 2. Amplitudes plotted against period for stars of groups 1 and 2. Filled circles represent group 1 stars, and triangles group 2 stars. Small symbols correspond to stars from Paper I and large symbols those from the present paper. Known type II Cepheids are plotted as open circles.

be an artifact of the data. In particular, long-term variations in the mean magnitude, footnote "d," are based on a comparison of magnitudes from the ASAS with those from Behlen. It is likely that many of the variations are real. However, there are indications that some of the ASAS magnitudes are affected by zero-point errors (for example, see the note for star A255 in the Appendix). Thus, the notes should be taken as suggestive of unstable variation that should be investigated further.

The comments in the Appendix include a number of possible cases of alternating cycles. This most commonly manifests itself as differences in the level of successive maxima but there are also stars with alternation in the minima, similar to what is seen in RV Tauri stars. We have listed the period between successive maxima or minima rather than the formal or double period 

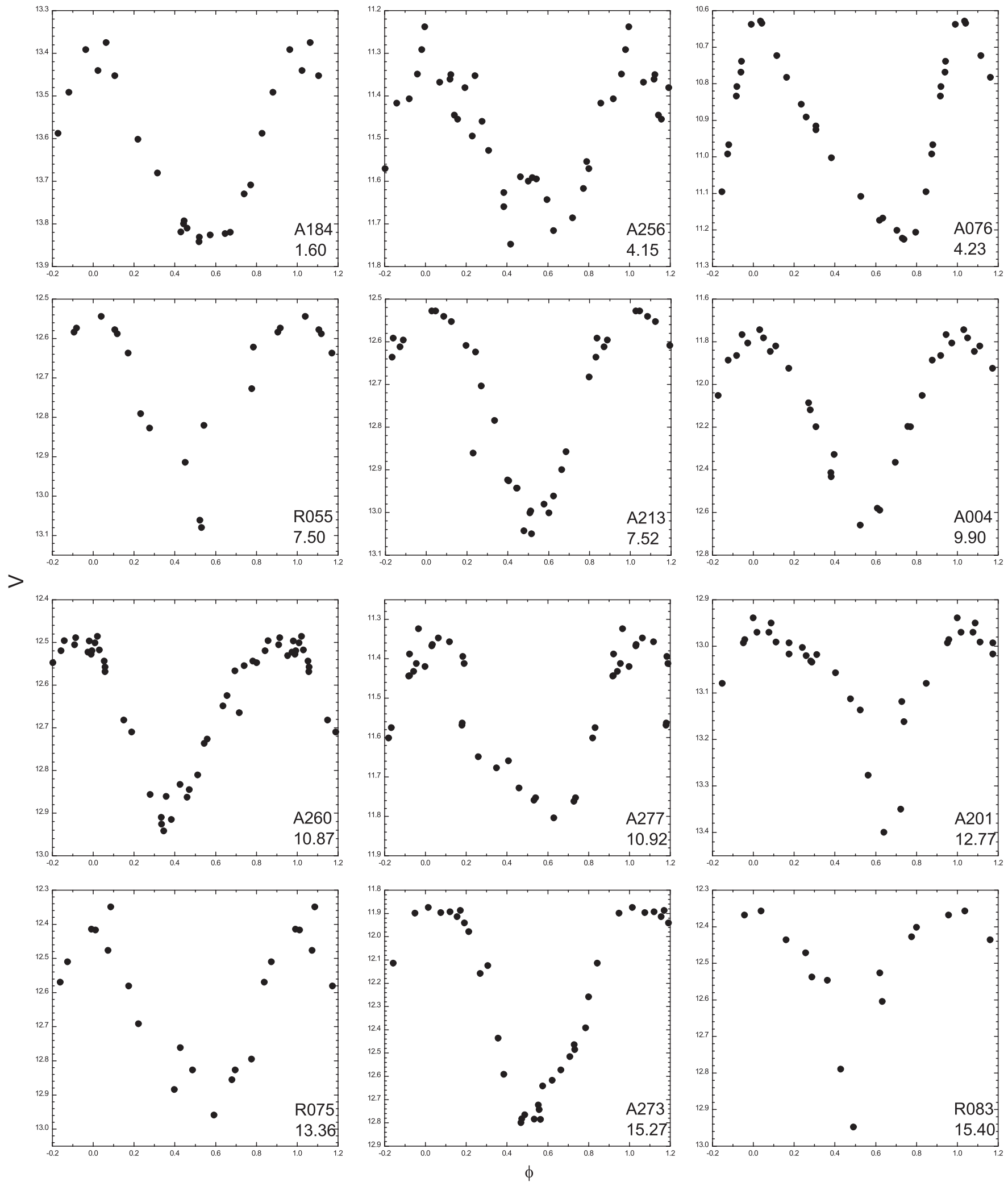

Figure 3. Light curves for the large amplitude Cepheid candidates belonging to group 1. Relative phases were calculated using periods from Table 3 and the points were arbitrarily shifted in phase to place maximum light at approximately zero.

given in the GCVS. We are unable to describe the variations of these stars in detail with the available data and they should be observed further. The periods cover a wide range from 2.3 days to 33 days. This argues against all of them being RV Tau stars.

\section{THE ANALYSIS OF THE OBSERVATIONS}

In Figure 1(a), we have plotted the mean colors against the periods of the stars that were not flagged as described in the previous section. Cepheid candidates from Paper I (identified as 

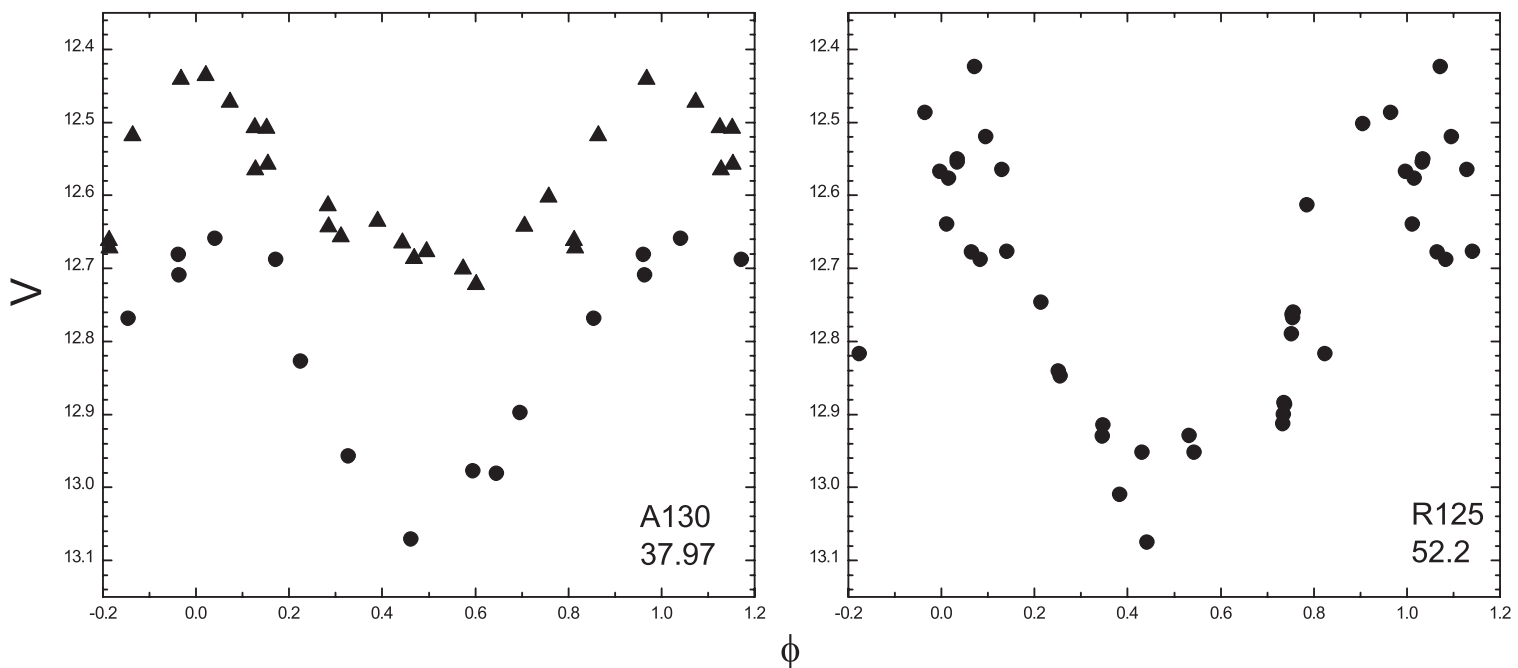

Figure 3. (Continued)
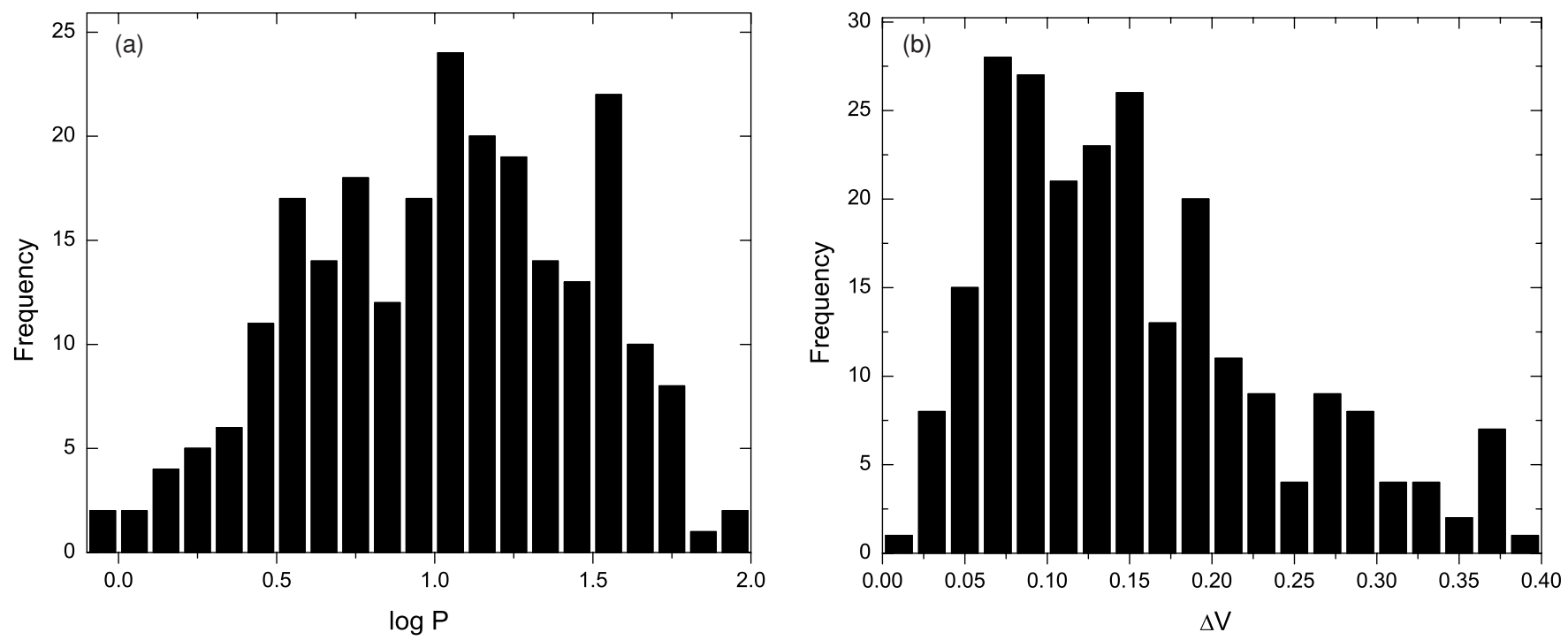

Figure 4. Distributions of the periods and amplitudes of the Cepheid-like stars from group 1.

status A or B there), stars from Paper I that were eliminated solely on the basis of red color and known Cepheids from Schmidt et al. (2004, 2005a, 2005b), are also shown.

For the purposes of the present discussion, we have grouped the stars according to where they fall in the diagram. Figure 1(b) shows these groupings and Column 13 of Table 3 lists the numbers of the groups to which individual stars belong. When the number is in parentheses it indicates that the star is just outside of the region, while a blank entry indicates stars which are not associated with any group or which were removed from the sample as discussed in the previous section.

\subsection{Group 1}

Group 1 coincides with the area occupied by the majority of the likely Cepheid candidates from Paper I. It should be noted that the delineated region is drawn rather arbitrarily and a number of stars just outside the boundary could justifiably have been included.

Although the boundaries of this region slope downward toward shorter periods, they might just as easily have been drawn level; there is little evidence that the color depends on period. Ignoring any period dependence, the spread of color among these stars is rather small; $\langle V-R\rangle=0.60 \pm 0.08$ (standard deviation). The narrow color range and the lack of period dependence contrasts with the known type II Cepheids and strongly suggests that these stars represent a different class of star, or at least a distinct subset of type II Cepheids. Spectra for the stars from Paper I support this conclusion (E. G. Schmidt et al. 2009, in preparation).

In Figure 2, we have plotted the amplitude against period for stars in groups 1 and 2. In Paper I, we considered stars with $\Delta V>0.40$ to be type II Cepheids. It can be seen that this is still reasonable with the larger sample.

Although we regard stars above this cutoff as very likely type II Cepheids, at lower amplitude the situation is unclear. The lack of small amplitudes among known type II Cepheids is certainly due in part to biases against the discovery of small amplitude variables. Thus, some type II Cepheids no doubt fall below the cutoff. Such stars cannot be reliably distinguished from the bulk of the small-amplitude stars based on photometry. We will refer to the small-amplitude stars as Cepheid-like stars. Spectra are being obtained to help distinguish bona fide small-amplitude type II Cepheids from the Cepheid-like stars. 

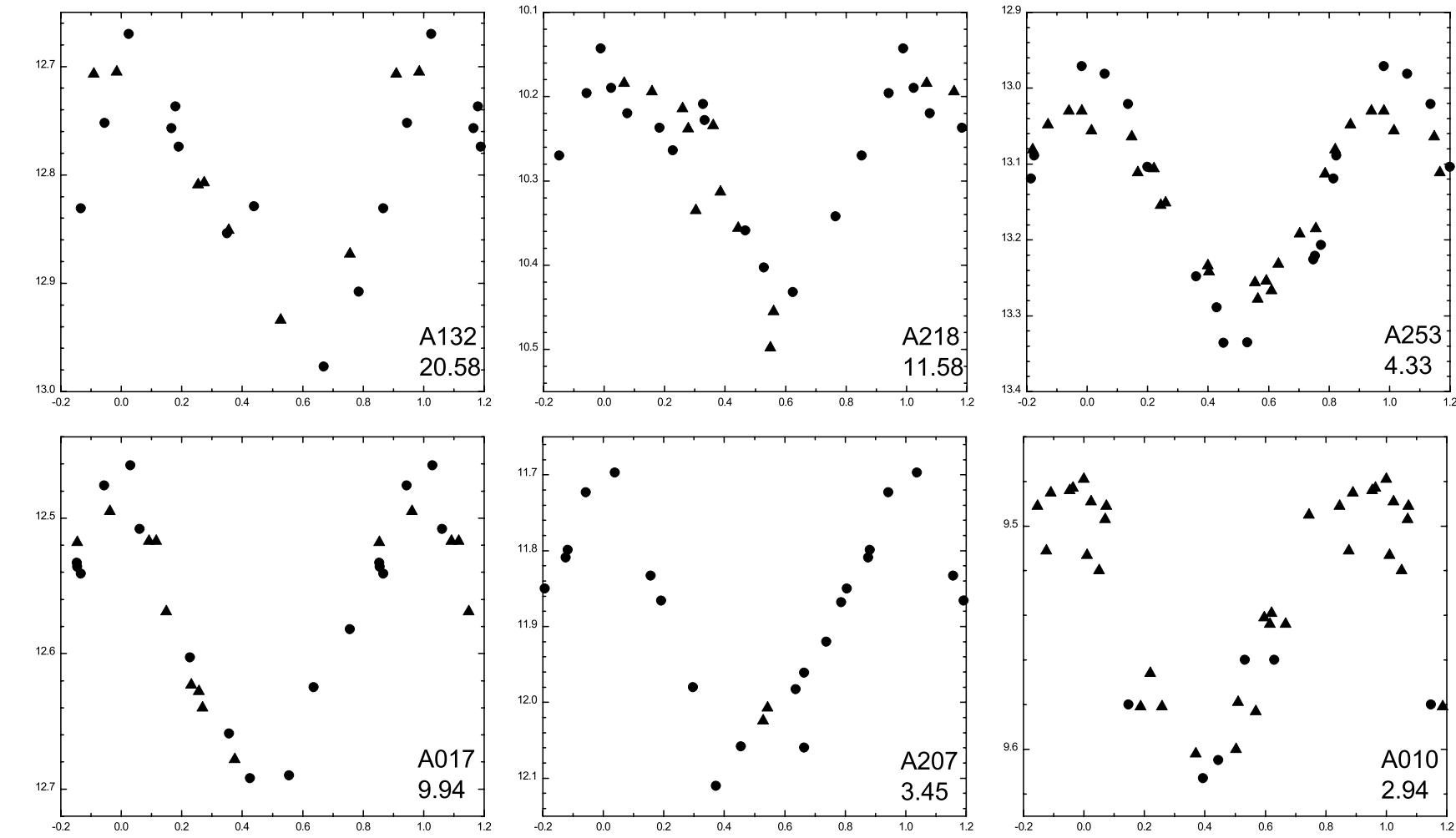

$>$
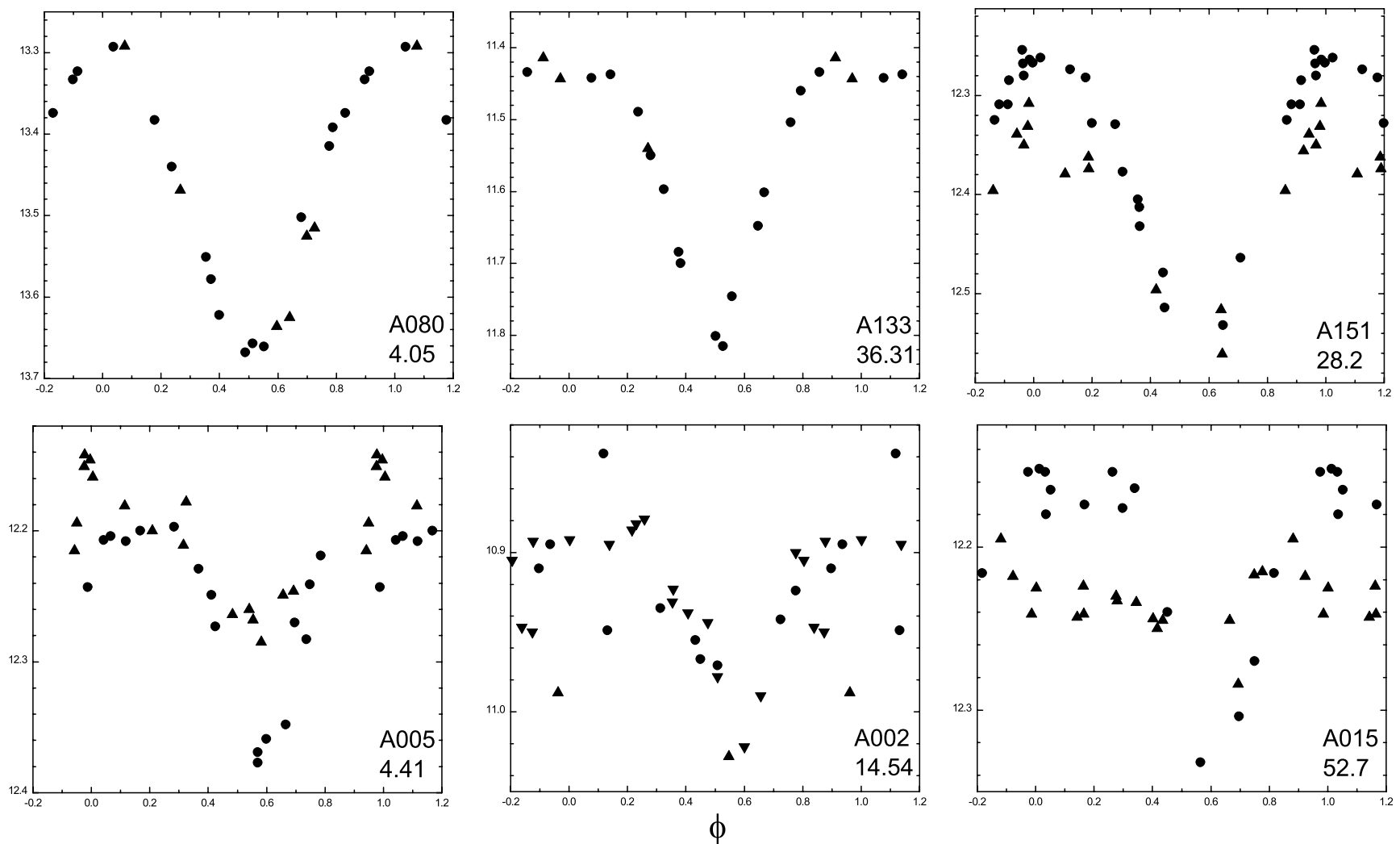

Figure 5. Light curves of representative Cepheid-like stars. Circles and triangles represent data from the first and second season of observation for each star.

There are 12 stars in region 1 which have amplitudes above the cutoff: A076, A130, A184, A201, A213, A256, A260, A273, and A277 from the present paper, and R055, R075, and R083 from Paper I. In addition, two large-amplitude stars fall just outside of the delineated area, A004, and R125. The light curves for these 14 stars are plotted in Figure 3.
A comparison of these light curves with those for known type II Cepheids of similar period (Schmidt et al. 2004, 2005a, 2005b) shows considerable similarity. Two exceptions may be A201 and R083. Both of these stars exhibit narrow minima that could easily be interpreted as eclipses. However, in both cases, the light curves vary considerably over a number of years and the 

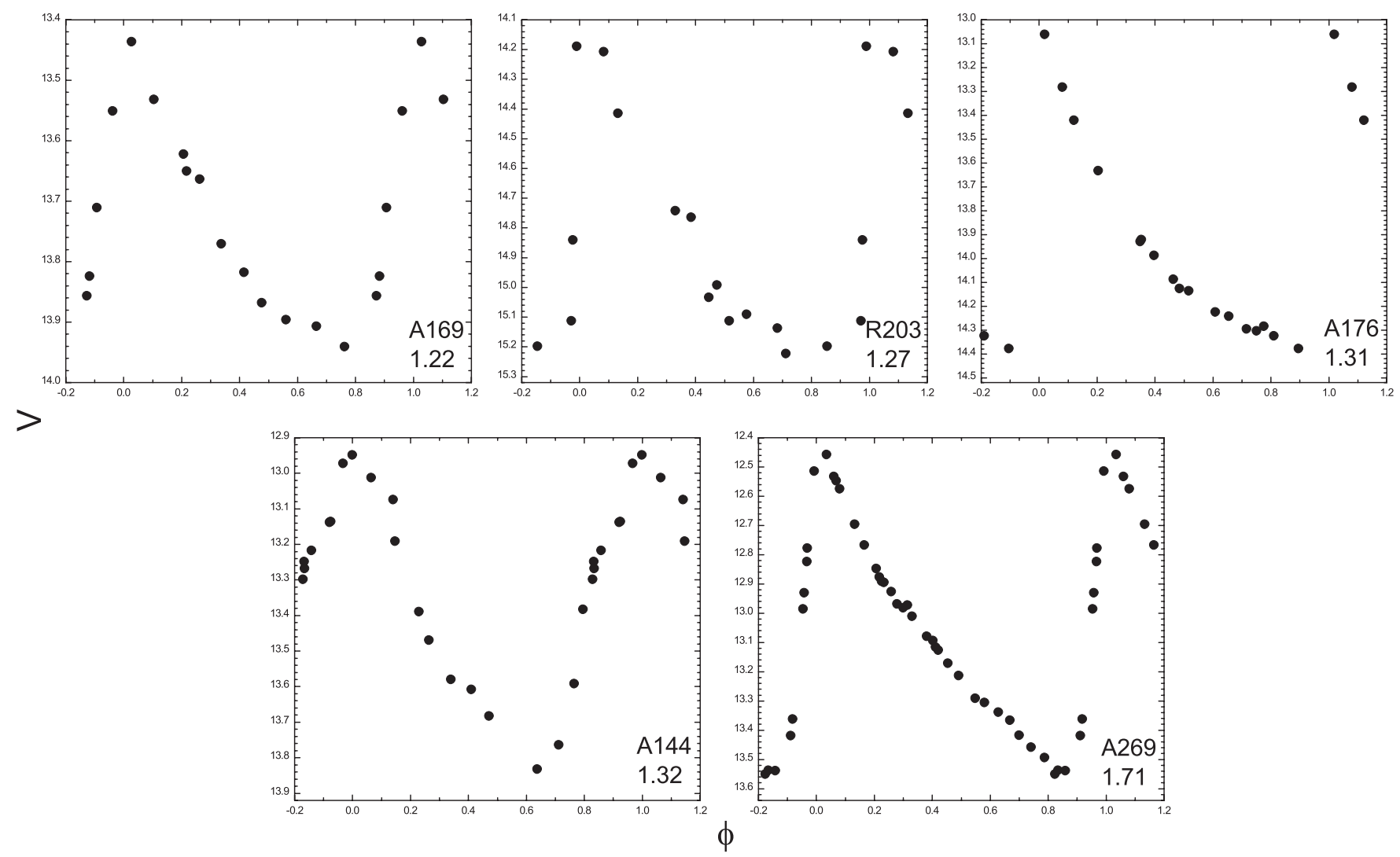

Figure 6. Light curves for the large amplitude Cepheid candidates belonging to group 2.

previous photometry fails to show any resemblance to eclipsing binary variations. Cepheid-like stars A005, A050, A133, A232, and A261 are similar cases. Rather flat maxima with narrow, often-symmetric minima are seen in a number of long-period type II Cepheids (e.g., PP Aql, and ET Vul; Schmidt et al. 2004). We apparently caught our seven stars during an interval when this behavior was extreme. This serves as a warning that light curves at a single epoch can be very misleading in the classification of variables. It is likely that further photometry of putative eclipsing stars from the sky surveys would uncover misclassified pulsating stars.

In Figure 4, we have plotted frequency distributions of the periods and amplitudes of the Cepheid-like stars from group 1 (i.e., all of the group 1 stars except those included in Figure 3). The period distribution lacks the gap between about 3 and 10 days ( $\log P=0.5-1.0$ ) that is a well-known feature of type II Cepheids (as compared to Figure 4(b) of Paper I). Furthermore, among type II Cepheids, there are approximately equal numbers of stars on the two sides of the gap. In Figure 4(a), there are only $22 \%$ as many short-period stars as long-period stars.

The distribution of amplitudes in Figure 4(b) peaks at about $0.1 \mathrm{mag}$. As noted above, we have no way to determine how many low-amplitude type II Cepheids are present in this plot. However, the rise in frequency toward lower amplitudes suggests that the sample is dominated by another type of object.

Footnotes "e," "h," "i," and "j" in Table 3 indicate period instability, while "c," "d," and "g" indicate long-term variations in amplitude, mean magnitude, or light curve shape. Just over $70 \%$ of the Cepheid-like stars have at least one of these footnotes. This is consistent with the known tendency of postasymptotic giant branch stars to exhibit unstable light curves (Kiss et al. 2007; Hrivnak et al. 2008) and provides support to the possibility that these stars are related to the type II Cepheids.

The light curves of the Cepheid-like stars exhibit a variety of forms. Many, especially those with small amplitudes, are fit adequately by a single sine curve. Others do not repeat well from cycle to cycle and sometimes the shape is quite different in different cycles. Hence, we only show a few examples in Figure 5 to illustrate the variety present. The first two light curves, for A132 and A218, have rising branches that are steeper than the declining branches. For the next two stars, A253 and A017, the light curves are more symmetric while A207 reverses the trend with a slower rise. The next four stars, A010, A080, A133, and A151, illustrate a common light curve form in which there is a relatively flat maximum with a narrow, symmetric minimum. This is similar to the type A light curves defined by Schmidt et al. (2004) for long-period type II Cepheids. As pointed out above, these light curves can be difficult to distinguish from those of eclipsing binaries. Finally, the last three stars in the figure possess light curves with considerable scatter due to nonrepetition between two seasons.

\subsection{Group 2}

Most of the known type II Cepheids with periods less than 2.2 days fall in this region along with 12 stars from the present sample. By contrast, only one Cepheid candidate from Paper I, R203, is found there.

Four of the present stars, A144, A169, A176, and A269, as well as R203 have amplitudes exceeding 0.4 mag. Their light curves are plotted in Figure 6. All except that of A144 are very asymmetric and resemble the light curves of type a RR Lyrae stars. Inspection of the light curves from Schmidt et al. (2005a) shows that this is common among short-period type II Cepheids. 
The light curve of A144 exhibits a slower rise with an inflection or small bump. This resembles the light curve of the known type II Cepheid V745 Oph. We conclude that these five stars are type II Cepheids.

The remaining eight stars have amplitudes less than 0.12 mag, considerably less than the large-amplitude stars. This argues that they are a separate type of object. Whether they are related to the Cepheid-like stars in group 1 is uncertain. Again, spectra will be obtained to clarify this question.

\subsection{Group 3}

In Paper I, we concluded that most of the stars in this region were red variables although some reddened Cepheids may also be found there. In the present paper, we have again found a significant number of such stars. However, it can be seen in Figure 1 that the present red stars occupy a significantly shorter period range than those from Paper I. The only other difference we can discern between the groups is that the red stars in the present sample are confined to amplitudes less than about 0.25 mag, while about a quarter of the stars from Paper I had larger amplitudes.

The surprising separation between the two samples might be explained by erroneous periods. In Paper I we determined periods from the NSVS and the Behlen data, while in the present paper we have also utilized the ASAS data. This should reduce aliasing in the present sample. To assess the importance of this effect, we redetermined periods for a number of stars using only the NSVS and Behlen data. By and large, the resulting periods were close to those given in Table 3. Thus, systematic period errors seem unlikely to explain the period separation.

The other possibility is that the identification or classification of variables differed in some way between the two surveys. This might also explain why group 2 is largely populated from the ASAS sample. The fact that some known large-amplitude stars are missing from the list of Akerlof et al. (2000) supports this supposition. Whatever the case, it raises a warning that we should treat samples derived from large-scale surveys with caution when completeness is important.

\subsection{Group 4}

Stars in this area have periods appropriate to short-period Cepheids but colors which are much redder. The largest amplitude among them is $\Delta V=0.16$. Although longer periods are possible for several stars (e.g., A052, A139, and ASA235), for most we can rule out a period long enough to move them into region 3. Again, most of these stars come from the present sample and we have no explanation for there being few such stars in the NSVS sample. We plan to obtain spectra of these stars to further explore their nature.

\section{CONCLUSIONS}

We have obtained photometry of 282 stars from the northern portion of the ASAS. We divided the stars into four groups based on location in the $\langle V-R\rangle$ versus period diagram. In the two groups that overlap the region of known type II Cepheids, we found 14 new type II Cepheids, bringing to 19 the number discovered in this program. The properties of the remaining stars are discussed. Their nature remains uncertain and spectra will be obtained to shed further light on them. Our results demonstrate serious biases in the identification of variable stars in large sky surveys such as the NSVS and the ASAS.
The research described here was based on the All Sky Automated Survey and utilized extensive data from it. The authors are grateful to Grzegorz Pojmanski and his collaborators for their hard work in producing the survey and for making the variable star lists and data available. This publication makes use of the data from the Northern Sky Variability Survey created jointly by the Los Alamos National Laboratory and the University of Michigan. The NSVS was funded by the Department of Energy, the National Aeronautics and Space Administration, and the National Science Foundation. Equipment used in the observations was purchased with funds from NSF grant AST 00-97353. We are grateful to the Department of Physics and Astronomy at the University of Nebraska for continued support for the operation of Behlen Observatory. D.R. and L.T.-L. were supported during part of this project by the Undergraduate Creative Activities and Research Experiences program at the University of Nebraska.

\section{APPENDIX}

A002: Possibly alternating cycles. See Figure 5.

A005: The photometric parameters in Table 3 are from the first season. During the second season $\langle V\rangle=12.28,\langle V-R\rangle=$ $0.65, \Delta V=0.11, \sigma_{V}=0.021$. The light curve of the first season resembles that of an eclipsing variable but the second season rules that out.

A006: Although the period of 23.56 fits all three data sets individually, the Behlen data are about 0.3 cycles out of phase relative to the others. This and several deviant points in the Behlen data suggest some irregularity in the pulsation.

A014: The tabulated period fits all of the data except the first season of the Behlen observations. The maximum of those data is offset from the other data sets by 0.4 cycles. The tabulated parameters refer to the second season. For the first season, $\langle V\rangle=10.06,\langle V-R\rangle=0.52, \Delta V=0.07, \sigma_{V}=0.012$.

A015: The period was determined using the NSVS and ASAS data. The photometric parameters were based on the first season of Behlen data. The Behlen data from the second season vary by no more than 0.03 mag with $\langle V\rangle=12.23,\langle V-R\rangle=0.55$. See Figure 5.

A018: Excess scatter around the phase of minimum light in all three data sets.

A021: Brighter at maximum and probably at minimum during the first year than during the second. Tabulated values were determined from all of the Behlen data. A026: The tabulated parameters refer to Behlen points before $\mathrm{HD}=2,453,992$. After $2,454,329\langle V\rangle=11.25,\langle V-R\rangle=0.62, \Delta V=0.03, \sigma_{V}=$ 0.006 .

A031: The mean magnitude, light curve shape, and amplitude vary from year to year. The mean magnitude and color and the scatter refer to all of the Behlen data, while the amplitude is from the interval from JD 2,453,711 to 2,453,797 when the amplitude was the largest for the Behlen data.

A034: The 1 day alias of the tabulated period, 0.94 days, is also possible but the longer period was selected since it is better for the Behlen data. There is possibly a long-term variation over several hundred days.

A038: After removal of six points during eclipse, a significant variation at about 0.14 days was found. This accounts for most of the scatter listed in Column 9. Although the most likely interpretation of the light curve is an eclipsing binary, a type II Cepheid or Cepheid-like star with a period of 9.124 days cannot be ruled out. 
A042: Low points in all three data sets suggest narrow eclipses. Except for the amplitude, the photometric parameters were determined with the low points omitted.

A044: The light curve shows strong modulation around the phase of maximum light, while the minima are relatively stable.

A050: The Behlen light curve resembles an eclipsing binary but this is ruled out by the earlier photometry.

A052: A period near 29 days is also possible.

A055: This star exhibits two types of behavior. At times, as during the second season of the Behlen observations, there is a regular periodic variation. At other times, as during the first and third seasons of the Behlen observations, no significant period could be found, the amplitude was smaller and the star was fainter by about $0.08 \mathrm{mag}$. The tabulated quantities were derived using only the second season of Behlen Observations.

A058: The 1 day alias of the tabulated period, $0.73879 \pm$ 0.00002 , is also possible.

A060: The scatter in the photometry suggests that this star may exhibit the Blazhko effect.

A068: The values in the table were determined from the second season of the Behlen data. There were too few data to determine the properties of the star during the first season but it is obviously fainter by about $0.05 \mathrm{mag}$.

A074: There is some evidence of alternating cycles, especially in the Behlen data. For this reason, the Fourier fit was done with a period of 4.727 days.

A077: It is possible that this star is a type II Cepheid with a period of 5.8304 days.

A084: A period of 0.86711 is also possible.

A085: The tabulated parameters refer to the interval from JD 2,454,365 to 2,454505. Earlier Behlen observations show negligible amplitude with $\langle V\rangle=9.29,\langle V-R\rangle=0.61, \sigma_{V}=$ 0.013 . The ASAS data also indicate a variable amplitude ranging from 0.03 to 0.09 over several seasons.

A090: The 1 day alias of the tabulated period, 1.03 days, is also possible.

A092: The NSVS data show small amplitude variation with a possible period of 23.5 days. Further observation would be worthwhile.

A093: The light curve and amplitude changed significantly between the first and the second seasons of the Behlen data. The tabulated parameters refer to the first season. During the second season, $\langle V\rangle=12.51,\langle V-R\rangle=0.61, \Delta V=0.12, \sigma_{V}=$ 0.033 .

A100: The 1 day alias of the tabulated period, 0.7456 days, cannot be excluded.

A111: A period of 21.3 fits the NSVS and ASAS data best. This is close to but, within the uncertainties, not exactly twice that of the Behlen photometry. This may be an example of period switching and merits further observation.

A112: Period obtained from the NSVS and ASAS data, 21 days, does not fit the Behlen data. Period scans on the latter produce many aliases. The tabulated period was arbitrarily selected as the closest to that of the NSVS and ASAS data.

A130: The light curve changed significantly between the first and second Behlen season and within the second season. The tabulated values refer to the first season. For the second season, they are $\langle V\rangle=12.59,\langle V-R\rangle=0.66, \Delta V=0.30, \sigma_{V}=$ 0.032 .

A133: Although the Behlen light curve resembles an eclipsing binary, the light curve from earlier photometry varies in shape and rules out such a star. See Figure 5.
A136: There is a possible alternation of the depth of the minima in the NSVS and ASAS data. The Behlen data are consistent with this but lacks the phase coverage to confirm it. The tabulated period refers to the time between successive maxima.

A139: A period of about 20 days cannot be excluded.

A146: The tabulated parameters were determined from the second season of data. During the first season, there was little variation and $\langle V\rangle=11.11,\langle V-R\rangle=1.01, \sigma_{V}=0.024$.

A147: A period near 44.05 days cannot be excluded.

A149: Alternation of cycles in the NSVS data.

A157: The 1 day alias of the tabulated period, 0.932 days, cannot be excluded.

A159: There were several low outliers in the Behlen data with no obvious explanation. Five obvious outliers were removed from the Behlen data and one from the ASAS data before the analysis.

A160: Possibly alternating cycles.

A173: The NSVS and ASAS magnitudes are about 0.9 mag brighter than the Behlen magnitudes. This is possibly due to the presence of a slightly brighter star $21^{\prime \prime}$ from the variable which may be included in the magnitude. There is some evidence of a systematic fading of the variable over the time span of the Behlen Observations. Two anomalous points were not included in determining the light-curve parameters. The NSVS and ASAS data indicate a period of 1.83 days.

A188: A period of 22.82 days is also possible. This is the 2 day alias of the adopted period.

A190: Only data from the second season were used to derive the photometric parameters. Only three points were obtained in the first season two of which differed from those of the second season by 0.05 mag.

A201: Although a narrow minimum in the Behlen light curve suggests an eclipsing binary, this is ruled out by the form of the earlier light curves, the variability of the light curve shape, and the variable period.

A204: The tabulated parameters were derived from the first season of Behlen observations when the pulsation appeared to be stable. At the beginning of the second season, the amplitude was about 0.18 mag but suddenly decreased to about 0.09 mag. The mean magnitude and color appeared to be unaffected.

A212: Little variation during the first season of Behlen observations. The tabulated period fits the NSVS and the second season of the Behlen observations. The amplitude and light curve scatter were derived from the second season of the Behlen data.

A214: No variation in NSVS data. Several other periods near 1 day are possible.

A216: NSVS photometry exhibited a period of apparent nonvariability. During times of variation, the maxima were offset by about 0.3 cycles from the ASAS and Behlen data.

A219: All three data sets have a small number of low points that suggest a narrow eclipse (about 0.04 cycles wide). Additionally, there is a modulation outside of eclipse which exhibits long-term changes in amplitude and mean magnitude. The tabulated parameters refer to the second Behlen season with the low points omitted. The first season gives $\langle V\rangle=$ $10.62,\langle V-R\rangle=0.59, \Delta V=0.09, \sigma_{V}=0.012$. The eclipse depths are about $0.5 \mathrm{mag}$.

A222: A period near 0.5 days is also possible. The adopted period was based on the second season of the ASAS data and the Behlen data. This star may exhibit alternation of cycles. 
A224: This star was essentially constant during the first season at $\langle V\rangle=9.50,\langle V-R\rangle=0.62, \sigma_{V}=0.012$. The tabulated parameters refer to the second season.

A228: A period of 0.898 days is also possible.

A229: In all three data sets $5 \%-10 \%$ of the data points near the phase of maximum light are about 0.08 mag brighter than the rest. The tabulated parameters were determined with the six such points in the Behlen data omitted. With those points the parameters become $\langle V\rangle=11.291,\langle V-R\rangle=0.275, \Delta V=$ $0.070, \sigma_{V}=0.029$.

A232: The Behlen light curves suggest the possibility that this star is an eclipsing binary but the earlier photometry rules this out.

A235: The parameters in the table refer to the second season of Behlen observations. For the first season, they are $\langle V\rangle=10.72,\langle V-R\rangle=0.55, \Delta V=0.24, \sigma_{V}=0.018$.

A238: Several possible periods near 1 day, although, the adopted period was slightly preferred. The parameters refer to the first season. During the second season, $\langle V\rangle=$ 11.65, $\langle V-R\rangle=0.50, \Delta V=0.03, \sigma_{V}=0.010$.

A240: A period of 1.3039 is also possible.

A251: Only the second season was used to determine the tabulated parameters. The variations during the first season may exhibit a slightly shorter period and $\langle V\rangle=11.40,\langle V-R\rangle=$ $0.72, \Delta V=0.18, \sigma_{V}=0.024$.

A255: Approximately half of the ASAS observations are offset nearly 0.2 mag brighter than the remainder of the ASAS observations and the Behlen Observations. Since they vary with the same period and since the offset observations are interleaved in time with those which are not offset, this is clearly an observational artifact.

A261: The Behlen light curve exhibits a narrow minimum which might indicate an eclipsing binary. However, this is ruled out by the NSVS and ASAS light curves.
A262: Strongly alternating depths of minima. The period refers to the interval between successive maxima.

A265: The parameters were determined from the second season of the Behlen data. During the first season, there was no significant variation and $\langle V\rangle=10.11,\langle V-R\rangle=0.43, \sigma_{V}=$ 0.011 .

A266: The light-curve parameters vary significantly from year to year. The values reported in the table refer to the first season of the Behlen data. The period is the best fit to the first season, while no satisfactory period could be found for the second season. For the second season, $\langle V\rangle=11.73,\langle V-R\rangle=$ $0.70, \Delta V=0.05, \sigma_{V}=0.028$.

A272: Large change in light curve between the first season and second season of Behlen data. The parameters in the table refer to the second season. For the first season, $\langle V\rangle=$ 11.60, $\langle V-R\rangle=0.60, \Delta V=0.18, \sigma_{V}=0.009$.

A279: There is a narrow eclipse and the period and amplitude refer to the eclipses. An additional long-term variation with an amplitude of several hundredths of a magnitude may be present out of eclipse.

\section{REFERENCES}

Akerlof, C., et al. 2000, AJ, 119, 1901

Ferraz-Mello, S. 1981, AJ, 86, 619

Hrivnak, B. J., Van Winckel, H., Reyniers, M., Bohlender, D., Waelkens, C., \& Lu, W. 2008, AJ, 136, 1557

Kiss, L. L., Derekas, A., Szabo, Gy. M., Bedding, T. R., \& Szabados, L. 2007, MNRAS, 375, 1338

Pojmanski, G., Pilecki, B., \& Szczygiel, D. 2005, Acta Astron, 55, 275

Schmidt, E. G., Johnston, D., Langan, S., \& Lee, K. M. 2004, AJ, 128, 1748

Schmidt, E. G., Johnston, D., Langan, S., \& Lee, K. M. 2005a, AJ, 129, 2007

Schmidt, E. G., Johnston, D., Langan, S., \& Lee, K. M. 2005b, AJ, 130, 832

Schmidt, E. G., Langan, S., Rogalla, D., \& Thacker-Lynn, L. 2007, AJ, 133, 665 (Paper I)

Stellingwerf, R. F. 1978, ApJ, 224, 953

Wozniak, P. R., et al. 2004, AJ, 127, 2436 